\title{
Self-fibrillating Cellulose Fibers: Rapid in situ Nanofibrillation to Prepare Strong, Transparent and Gas Barrier Nanopapers
}

Yunus Can Gorur, Per A. Larsson, Lars Wågberg*

Fibre and Polymer Technology, KTH Royal Institute of Technology, SE-10044 Stockholm, Sweden

*Corresponding author: wagberg@kth.se 


\section{Supporting Information}

Table S1. Crystallinity index and crystallite size values of unmodified fibers (Reference) and SFFs before and after fibrillation.

\begin{tabular}{lcccc}
\hline & Crystallinity (\%) & \multicolumn{3}{c}{ Crystallite Size (nm) } \\
& & $\mathbf{( 1 0 1 )}$ & $\mathbf{( 1 0 1})$ & $(\mathbf{0 0 2})$ \\
\hline Reference & 78 & 2.3 & 2.4 & 4.2 \\
SFFs (Unfibrillated) & 75 & 2.1 & 2.2 & 3.6 \\
SFFs (Fibrillated) & 75 & 2.1 & 2.2 & 3.6 \\
\hline
\end{tabular}

Table S2. Optical properties of SFF papers before and after fibrillation. Transmittance and haze data are given at a wavelength of $600 \mathrm{~nm}$.

\begin{tabular}{lccc}
\hline Sample Name & Thickness $(\boldsymbol{\mu m})$ & Transmittance (\%) & Haze (\%) \\
\hline SFF at $\mathrm{pH} 2$ & 105 & 83 & 71 \\
SFF at $\mathrm{pH} 12$ & 105 & 87 & 47 \\
\hline
\end{tabular}

